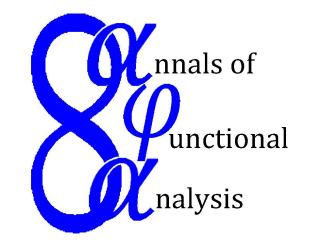

Ann. Funct. Anal. 6 (2015), no. 4, 289-300

http://doi.org/10.15352/afa/06-4-289

ISSN: 2008-8752 (electronic)

http://projecteuclid.org/afa

\title{
SHANNON TYPE INEQUALITIES OF A RELATIVE OPERATOR ENTROPY INCLUDING TSALLIS AND RÉNYI ONES
}

\author{
HIROSHI ISA ${ }^{1}$, MASATOSHI ITO $^{1 *}$, EIZABURO KAMEI ${ }^{2}$, HIROAKI TOHYAMA ${ }^{1}$ AND \\ MASAYUKI WATANABE ${ }^{1}$ \\ Communicated by Y. Seo
}

Abstract. Let $\mathbb{A}=\left(A_{1}, \cdots, A_{n}\right)$ and $\mathbb{B}=\left(B_{1}, \cdots, B_{n}\right)$ be operator distributions, that is, $A_{i}, B_{i}>0(1 \leq i \leq n)$ and $\sum_{i=1}^{n} A_{i}=\sum_{i=1}^{n} B_{i}=I$. We give a new relative operator entropy of two operator distributions as follows: For $t, s \in \mathbb{R} \backslash\{0\}$,

$$
K_{t, s}(\mathbb{A} \mid \mathbb{B}) \equiv \frac{\left(\sum_{i=1}^{n} A_{i} \natural_{t} B_{i}\right)^{s}-I}{t s}
$$

where $A \natural_{t} B=A^{\frac{1}{2}}\left(A^{\frac{-1}{2}} B A^{\frac{-1}{2}}\right)^{t} A^{\frac{1}{2}}$. This includes relative operator entropy $S(\mathbb{A} \mid \mathbb{B})$, Rényi relative operator entropy $I_{t}(\mathbb{A} \mid \mathbb{B})$ and Tsallis relative operator entropy $T_{t}(\mathbb{A} \mid \mathbb{B})$.

In this paper, firstly, we discuss fundamental properties of $K_{t, s}(\mathbb{A} \mid \mathbb{B})$. Secondly, we obtain Shannon type operator inequalities by using $K_{t, s}(\mathbb{A} \mid \mathbb{B})$, which include previous results by Furuta, Yanagi-Kuriyama-Furuichi and ourselves.

\section{INTRODUCTION}

In this paper, an operator means a bounded linear operator on a Hilbert space $\mathcal{H}$. An operator $T$ is said to be positive (denoted by $T \geq 0$ ) if $(T x, x) \geq 0$ for all $x \in \mathcal{H}$, and also an operator $T$ is said to be strictly positive (denoted by $T>0$ ) if $T$ is positive and invertible.

Date: Received: May 2, 2015; Accepted: Jun. 18, 2015.

* Corresponding author.

2010 Mathematics Subject Classification. Primary 47A63; Secondary 47A64, 94A17.

Key words and phrases. Tsallis relative operator entropy, operator Shannon inequality, Rényi relative operator entropy, Shannon-Nakamura-Umegaki entropy. 
For $A, B>0$, we consider a path passing through $A$ and $B$ as follows: $A \natural_{t} B=$ $A^{\frac{1}{2}}\left(A^{\frac{-1}{2}} B A^{\frac{-1}{2}}\right)^{t} A^{\frac{1}{2}}$ for $t \in \mathbb{R}$. We remark that for $0 \leq t \leq 1, A \natural_{t} B$ is known as the weighted geometric mean, which is denoted by $A \sharp_{t} B$.

For discrete probability distributions $p=\left(p_{1}, p_{2}, \cdots, p_{n}\right)$ and $q=\left(q_{1}, q_{2}, \cdots, q_{n}\right)$, that is, $p_{i}, q_{i}>0(1 \leq i \leq n)$ and $\sum_{i=1}^{n} p_{i}=\sum_{i=1}^{n} q_{i}=1$, the relative entropy (Kullback-Leibler divergence, Kullback-Leibler distance) is defined by $s(p \mid q) \equiv \sum_{i=1}^{n} p_{i} \log \frac{p_{i}}{q_{i}}([12,14])$. We remark that if $q=\left(\frac{1}{n}, \frac{1}{n}, \ldots, \frac{1}{n}\right)$, then $s(p \mid q)=\log n-h(p)$, where $h(p) \equiv-\sum_{i=1}^{n} p_{i} \log p_{i}$ is the famous Shannon entropy. The relative entropy plays an important role in the classical information theory as a notion to measure the difference between two probability distributions. We also remark that $s(p \mid q) \geq 0$ holds for any probability distributions $p$ and $q$, which is equivalent to Shannon inequality (Shannon lemma, Gibbs' inequality) $-\sum_{i=1}^{n} p_{i} \log p_{i} \leq-\sum_{i=1}^{n} p_{i} \log q_{i}$.

As an operator version of $s(p \mid q)$, for $A, B>0$, relative operator entropy

$$
S(A \mid B) \equiv A^{\frac{1}{2}} \log \left(A^{\frac{-1}{2}} B A^{\frac{-1}{2}}\right) A^{\frac{1}{2}}
$$

is introduced in [4], and Furuta [2] introduced its generalization

$$
S_{t}(A \mid B) \equiv A^{\frac{1}{2}}\left(A^{\frac{-1}{2}} B A^{\frac{-1}{2}}\right)^{t} \log \left(A^{\frac{-1}{2}} B A^{\frac{-1}{2}}\right) A^{\frac{1}{2}}
$$

for $t \in \mathbb{R}$. We remark that $S(A \mid I)=-A \log A$ is operator entropy given by Nakamura-Umegaki [13], and we denote $S(A \mid I)$ by $H(A) . \quad S_{t}(A \mid B)$ can be considered as a tangent at $t$ of $A \natural_{t} B$. If $t=0$, then $S(A \mid B)=S_{0}(A \mid B)$. Yanagi-Kuriyama-Furuichi introduced Tsallis relative operator entropy in [16]. For $A, B>0$ and $t \in \mathbb{R}$, Tsallis relative operator entropy is defined by

$$
\begin{aligned}
& T_{t}(A \mid B) \equiv \frac{A^{\frac{1}{2}}\left(A^{\frac{-1}{2}} B A^{\frac{-1}{2}}\right)^{t} A^{\frac{1}{2}}-A}{t}=\frac{A \natural_{t} B-A}{t} \quad(t \neq 0), \\
& T_{0}(A \mid B) \equiv \lim _{t \rightarrow 0} T_{t}(A \mid B)=S(A \mid B) .
\end{aligned}
$$

We call an operator sequence $\mathbb{A}=\left(A_{1}, \cdots, A_{n}\right)$ operator distribution if $A_{i}>0$ $(1 \leq i \leq n)$ and $\sum_{i=1}^{n} A_{i}=I$, since it can be regarded as an operator version of discrete probability distribution. We remark that operator distribution is called POVM (positive operator valued measure) in quantum information theory. In $[8,10]$, we define relative operator entropy $S(\mathbb{A} \mid \mathbb{B})$, generalized relative operator entropy $S_{t}(\mathbb{A} \mid \mathbb{B})$, Tsallis relative operator entropy $T_{t}(\mathbb{A} \mid \mathbb{B})$ and Rényi relative operator entropy $I_{t}(\mathbb{A} \mid \mathbb{B})$ of two operator distributions $\mathbb{A}=\left(A_{1}, \cdots, A_{n}\right)$ and $\mathbb{B}=\left(B_{1}, \cdots, B_{n}\right)$ as follows: For $t \in \mathbb{R}$,

$$
\begin{aligned}
S(\mathbb{A} \mid \mathbb{B}) & \equiv \sum_{i=1}^{n} S\left(A_{i} \mid B_{i}\right), \quad S_{t}(\mathbb{A} \mid \mathbb{B}) \equiv \sum_{i=1}^{n} S_{t}\left(A_{i} \mid B_{i}\right), \\
T_{t}(\mathbb{A} \mid \mathbb{B}) & \equiv \sum_{i=1}^{n} T_{t}\left(A_{i} \mid B_{i}\right), \\
I_{t}(\mathbb{A} \mid \mathbb{B}) & \equiv \frac{1}{t} \log \sum_{i=1}^{n} A_{i} \natural_{t} B_{i} \quad(\text { if } t \neq 0) \quad \text { and } I_{0}(\mathbb{A} \mid \mathbb{B}) \equiv \lim _{t \rightarrow 0} I_{t}(\mathbb{A} \mid \mathbb{B}) .
\end{aligned}
$$


On the other hand, for two operator distributions $\mathbb{A}=\left(A_{1}, \cdots, A_{n}\right)$ and $\mathbb{B}=$ $\left(B_{1}, \cdots, B_{n}\right)$, Furuta [2] had shown an operator version of Shannon inequality (briefly, operator Shannon inequality) and its reverse one.

$$
-\log \left[\sum_{i=1}^{n} A_{i} B_{i}^{-1} A_{i}\right] \leq S(\mathbb{A} \mid \mathbb{B}) \leq 0
$$

Yanagi-Kuriyama-Furuichi [16] showed a substitution of (1.1) by using Tsallis relative operator entropy.

$$
\frac{\left(\sum_{i=1}^{n} A_{i} B_{i}^{-1} A_{i}\right)^{-t}-I}{t} \leq T_{t}(\mathbb{A} \mid \mathbb{B}) \leq 0 \quad \text { for } 0<t \leq 1
$$

Recently, as an extension of operator Shannon inequality, we obtained

$$
\begin{gathered}
S(\mathbb{A} \mid \mathbb{B}) \leq I_{t}(\mathbb{A} \mid \mathbb{B}) \leq T_{t}(\mathbb{A} \mid \mathbb{B}) \leq 0 \\
0 \leq-T_{1-t}(\mathbb{B} \mid \mathbb{A}) \leq-I_{1-t}(\mathbb{B} \mid \mathbb{A}) \leq S_{1}(\mathbb{A} \mid \mathbb{B}) \\
T_{t}(\mathbb{A} \mid \mathbb{B}) \leq S_{t}(\mathbb{A} \mid \mathbb{B}) \leq-T_{1-t}(\mathbb{B} \mid \mathbb{A})
\end{gathered}
$$

for $0<t<1$ in [8].

In [10] (see also [9]), we introduced generalizations of $S(\mathbb{A} \mid \mathbb{B}), I_{t}(\mathbb{A} \mid \mathbb{B})$ and $T_{t}(\mathbb{A} \mid \mathbb{B})$ by using power mean, and we obtain generalizations of (1.3), (1.4) and $(1.5)$.

Here, we introduce a new operator entropy including $T_{t}(\mathbb{A} \mid \mathbb{B})$ and $I_{t}(\mathbb{A} \mid \mathbb{B})$ as a different generalization from [10].

Definition 1. Let $\mathbb{A}=\left(A_{1}, \cdots, A_{n}\right)$ and $\mathbb{B}=\left(B_{1}, \cdots, B_{n}\right)$ be operator distributions. For $t, s \in \mathbb{R} \backslash\{0\}$,

$$
K_{t, s}(\mathbb{A} \mid \mathbb{B}) \equiv \frac{\left(\sum_{i=1}^{n} A_{i} \natural_{t} B_{i}\right)^{s}-I}{t s} .
$$

We define $K_{t, 0}(\mathbb{A} \mid \mathbb{B})$ and $K_{0, s}(\mathbb{A} \mid \mathbb{B})$ by the limit of $K_{t, s}(\mathbb{A} \mid \mathbb{B})$ as a parameter tends to 0 , respectively.

In this paper, firstly, we discuss fundamental properties of $K_{t, s}(\mathbb{A} \mid \mathbb{B})$. Secondly, we obtain Shannon type operator inequalities by using $K_{t, s}(\mathbb{A} \mid \mathbb{B})$, which include (1.1), (1.2) and (1.3). Lastly, we introduce Shannon-Nakamura-Umegaki operator entropy and give fundamental properties. 


\section{Properties of $K_{t, s}(\mathbb{A} \mid \mathbb{B})$}

First, we state relations among $T_{t}(\mathbb{A} \mid \mathbb{B}), I_{t}(\mathbb{A} \mid \mathbb{B})$ and $K_{t, s}(\mathbb{A} \mid \mathbb{B})$.

Proposition 2.1. Let $\mathbb{A}=\left(A_{1}, \cdots, A_{n}\right)$ and $\mathbb{B}=\left(B_{1}, \cdots, B_{n}\right)$ be operator distributions.

(i) $K_{t, 0}(\mathbb{A} \mid \mathbb{B})=I_{t}(\mathbb{A} \mid \mathbb{B})$ for $t \in \mathbb{R} \backslash\{0\}$.

(ii) $K_{t, 1}(\mathbb{A} \mid \mathbb{B})=T_{t}(\mathbb{A} \mid \mathbb{B})$ for $t \in \mathbb{R} \backslash\{0\}$.

(iii) For $t>0$ and $0<s<1, S(\mathbb{A} \mid \mathbb{B}) \leq I_{t}(\mathbb{A} \mid \mathbb{B}) \leq K_{t, s}(\mathbb{A} \mid \mathbb{B}) \leq T_{t}(\mathbb{A} \mid \mathbb{B})$.

(iv) For $t<0$ and $0<s<1$, $T_{t}(\mathbb{A} \mid \mathbb{B}) \leq K_{t, s}(\mathbb{A} \mid \mathbb{B}) \leq I_{t}(\mathbb{A} \mid \mathbb{B}) \leq S(\mathbb{A} \mid \mathbb{B})$.

If $0<t \leq 1$, then (iii) in Proposition 2.1 ensures

$$
S(\mathbb{A} \mid \mathbb{B}) \leq I_{t}(\mathbb{A} \mid \mathbb{B}) \leq K_{t, s}(\mathbb{A} \mid \mathbb{B}) \leq T_{t}(\mathbb{A} \mid \mathbb{B}) \leq 0
$$

by (1.2), so that (2.1) is an improvement of (1.3) by using $K_{t, s}(\mathbb{A} \mid \mathbb{B})$.

Jensen's operator inequality [7] plays an important role to prove our results.

Theorem 2.A (Jensen's operator inequality [7]). Let $f(x)$ be an operator concave function on an interval $J$. Let $\left\{C_{i}\right\}_{i=1}^{n}$ be operators with $\sum_{i=1}^{n} C_{i}^{*} C_{i}=I$. Then

$$
f\left(\sum_{i=1}^{n} C_{i}^{*} A_{i} C_{i}\right) \geq \sum_{i=1}^{n} C_{i}^{*} f\left(A_{i}\right) C_{i}
$$

holds for every selfadjoint operators $\left\{A_{i}\right\}_{i=1}^{n}$ whose spectra are contained in $J$.

Proof of Proposition 2.1. (i) Since $\lim _{s \rightarrow 0} \frac{a^{s}-1}{s}=\log a$ for $a>0$, we have

$$
K_{t, 0}(\mathbb{A} \mid \mathbb{B})=\frac{1}{t} \lim _{s \rightarrow 0} \frac{\left(\sum_{i=1}^{n} A_{i} \natural_{t} B_{i}\right)^{s}-I}{s}=\frac{1}{t} \log \sum_{i=1}^{n} A_{i} \natural_{t} B_{i}=I_{t}(\mathbb{A} \mid \mathbb{B}) .
$$

(ii) We obtain (ii) as follows:

$$
K_{t, 1}(\mathbb{A} \mid \mathbb{B})=\frac{\sum_{i=1}^{n} A_{i} \natural_{t} B_{i}-I}{t}=\sum_{i=1}^{n} \frac{A_{i} \natural_{t} B_{i}-A_{i}}{t}=T_{t}(\mathbb{A} \mid \mathbb{B}) .
$$

(iii) Since $\log a \leq \frac{a^{s}-1}{s} \leq a-1$ holds for $a>0$ and $0<s<1$,

$$
\frac{1}{t} \log \sum_{i=1}^{n} A_{i} \natural_{t} B_{i} \leq \frac{\left(\sum_{i=1}^{n} A_{i} \natural_{t} B_{i}\right)^{s}-I}{s t} \leq \frac{\sum_{i=1}^{n} A_{i} \natural_{t} B_{i}-I}{t},
$$

that is, $I_{t}(\mathbb{A} \mid \mathbb{B}) \leq K_{t, s}(\mathbb{A} \mid \mathbb{B}) \leq T_{t}(\mathbb{A} \mid \mathbb{B})$ holds for $t>0$ and $0<s<1$. The relation $S(\mathbb{A} \mid \mathbb{B}) \leq I_{t}(\mathbb{A} \mid \mathbb{B})$ has already shown in (1.3).

(iv) By the similar way to the proof of (iii), we have $T_{t}(\mathbb{A} \mid \mathbb{B}) \leq K_{t, s}(\mathbb{A} \mid \mathbb{B}) \leq$ $I_{t}(\mathbb{A} \mid \mathbb{B})$ for $t<0$ and $0<s<1$. 
Since $f(x)=\log x$ is operator concave for $x>0$, we have

$$
\begin{aligned}
I_{t}(\mathbb{A} \mid \mathbb{B}) & =\frac{1}{t} \log \sum_{i=1}^{n} A_{i}^{\frac{1}{2}}\left(A_{i}^{\frac{-1}{2}} B_{i} A_{i}^{\frac{-1}{2}}\right)^{t} A_{i}^{\frac{1}{2}} \\
& \leq \frac{1}{t} \sum_{i=1}^{n} A_{i}^{\frac{1}{2}} \log \left(A_{i}^{\frac{-1}{2}} B_{i} A_{i}^{\frac{-1}{2}}\right)^{t} A_{i}^{\frac{1}{2}} \\
& =\sum_{i=1}^{n} A_{i}^{\frac{1}{2}} \log \left(A_{i}^{\frac{-1}{2}} B_{i} A_{i}^{\frac{-1}{2}}\right) A_{i}^{\frac{1}{2}}=S(\mathbb{A} \mid \mathbb{B}) .
\end{aligned}
$$

for $t<0$ by using Theorem 2.A. Hence the proof is complete.

Remark. We remark that $I_{0}(\mathbb{A} \mid \mathbb{B}) \equiv \lim _{t \rightarrow 0} I_{t}(\mathbb{A} \mid \mathbb{B})=S(\mathbb{A} \mid \mathbb{B})$ holds by (iii) and (iv) in Proposition 2.1.

Next, we discuss monotonicity of $K_{t, s}(\mathbb{A} \mid \mathbb{B})$ on $s$.

Proposition 2.2. Let $\mathbb{A}=\left(A_{1}, \cdots, A_{n}\right)$ and $\mathbb{B}=\left(B_{1}, \cdots, B_{n}\right)$ be operator distributions.

(i) For $t>0, K_{t, s}(\mathbb{A} \mid \mathbb{B})$ is increasing for $s \in \mathbb{R}$.

(ii) For $t<0, K_{t, s}(\mathbb{A} \mid \mathbb{B})$ is decreasing for $s \in \mathbb{R}$.

(iii) $K_{0, s}(\mathbb{A} \mid \mathbb{B})=S(\mathbb{A} \mid \mathbb{B})$ for $s \in \mathbb{R} \backslash\{0\}$.

Proof. We have (i) and (ii) immediately since for $a>0$,

$$
f(s)= \begin{cases}\frac{a^{s}-1}{s} & (s \neq 0) \\ \log a & (s=0)\end{cases}
$$

is increasing for $s \in \mathbb{R}$.

We prove (iii) as follows: For each $s \in \mathbb{R} \backslash\{0\}$, there exists a natural number $m$ such that $|s| \leq m$. Therefore, if $t>0$, then

$$
K_{t,-m}(\mathbb{A} \mid \mathbb{B}) \leq K_{t, s}(\mathbb{A} \mid \mathbb{B}) \leq K_{t, m}(\mathbb{A} \mid \mathbb{B})
$$

holds by (i). Similarly, if $t<0$, then

$$
K_{t, m}(\mathbb{A} \mid \mathbb{B}) \leq K_{t, s}(\mathbb{A} \mid \mathbb{B}) \leq K_{t,-m}(\mathbb{A} \mid \mathbb{B})
$$

holds by (ii).

Put $X_{t}=\sum_{i=1}^{n} A_{i} \natural_{t} B_{i}$. Then, since $X_{0}=I$, we have

$$
\begin{aligned}
K_{t, m}(\mathbb{A} \mid \mathbb{B}) & =\frac{X_{t}^{m}-I}{t m}=\frac{\left(X_{t}-I\right)\left(X_{t}^{m-1}+X_{t}^{m-2}+\cdots+I\right)}{t m} \\
& =T_{t}(\mathbb{A} \mid \mathbb{B}) \cdot \frac{X_{t}^{m-1}+X_{t}^{m-2}+\cdots+I}{m} \rightarrow S(\mathbb{A} \mid \mathbb{B}) \quad \text { as } t \rightarrow 0
\end{aligned}
$$


and

$$
K_{t,-m}(\mathbb{A} \mid \mathbb{B})=\frac{X_{t}^{-m}-I}{-t m}=X_{t}^{-m} \cdot \frac{X_{t}^{m}-I}{t m} \rightarrow S(\mathbb{A} \mid \mathbb{B}) \quad \text { as } t \rightarrow 0 .
$$

Therefore we obtain $\lim _{t \rightarrow 0} K_{t, s}(\mathbb{A} \mid \mathbb{B})=S(\mathbb{A} \mid \mathbb{B})$ by $(2.2)$ and $(2.3)$.

Remark. The same argument to the proof of (iii) in Proposition 2.2 yields

$$
K_{0,0}(\mathbb{A} \mid \mathbb{B}) \equiv \lim _{(t, s) \rightarrow(0,0)} K_{t, s}(\mathbb{A} \mid \mathbb{B})=S(\mathbb{A} \mid \mathbb{B}),
$$

so we can allow the case $t=0$ in (i) and (ii) in Proposition 2.1, and the case $s=0$ in (iii) in Proposition 2.2.

When we consider monotonicity of $K_{t, s}(\mathbb{A} \mid \mathbb{B})$ on $t$, we might want to put $s=\frac{\beta}{t}$. Then we have the following result on monotonicity of $K_{t, \frac{\beta}{t}}(\mathbb{A} \mid \mathbb{B})$ on $t$.

Theorem 2.3. Let $\mathbb{A}=\left(A_{1}, \cdots, A_{n}\right)$ and $\mathbb{B}=\left(B_{1}, \cdots, B_{n}\right)$ be operator distributions.

(i) For $\beta>0, K_{t, \frac{\beta}{t}}(\mathbb{A} \mid \mathbb{B})$ is increasing for $t$ such that $t \leq-\beta$ or $\beta \leq t$.

(ii) For $\beta>0, K_{t, \frac{-\beta}{t}}(\mathbb{A} \mid \mathbb{B})$ is increasing for $t$ such that $t \leq-\beta$ or $\beta \leq t$.

(iii) $I_{t}(\mathbb{A} \mid \mathbb{B})$ is increasing for $t \in \mathbb{R}$.

In order to prove Theorem 2.3, we use the following lemma.

Lemma 2.4. Let $\left\{C_{i}\right\}_{i=1}^{n}$ be invertible operators with $\sum_{i=1}^{n} C_{i}^{*} C_{i}=I$ and $\left\{X_{i}\right\}_{i=1}^{n}$ be strictly positive operators.

(i) For a fixed $\beta>0$, the function $g_{1}(\lambda)=\left(\sum_{i=1}^{n} C_{i}^{*} X_{i}^{\lambda} C_{i}\right)^{\frac{\beta}{\lambda}}$ defined on $J \equiv(-\infty,-\beta] \cup[\beta, \infty)$ is increasing on the interval $J$.

(ii) The function $g_{2}(\lambda)=\frac{1}{\lambda} \log \left(\sum_{i=1}^{n} C_{i}^{*} X_{i}^{\lambda} C_{i}\right)$ is increasing for $\lambda \in \mathbb{R}$, where $g_{2}(0) \equiv \sum_{i=1}^{n} C_{i}^{*}\left(\log X_{i}\right) C_{i}$.

Proof. (i) By Theorem 2.A,

$$
\left(\sum_{i=1}^{n} C_{i}^{*} X_{i}^{\lambda} C_{i}\right)^{\frac{\mu}{\lambda}} \geq \sum_{i=1}^{n} C_{i}^{*} X_{i}^{\mu} C_{i} \quad \text { for } 0<\mu \leq \lambda
$$


since $f(x)=x^{\frac{\mu}{\lambda}}$ is operator concave. Applying Loewner-Heinz theorem " $A \geq$ $B \geq 0$ ensures $A^{\alpha} \geq B^{\alpha}$ for any $\alpha \in[0,1]$ " to (2.4), we have

$$
g_{1}(\mu)=\left(\sum_{i=1}^{n} C_{i}^{*} X_{i}^{\mu} C_{i}\right)^{\frac{\beta}{\mu}} \leq\left(\sum_{i=1}^{n} C_{i}^{*} X_{i}^{\lambda} C_{i}\right)^{\frac{\beta}{\lambda}}=g_{1}(\lambda)
$$

for $0<\beta \leq \mu \leq \lambda$. Replacing $X_{i}$ by $X_{i}^{-1}$ and taking inverses to (2.5), we have

$$
g_{1}(-\lambda)=\left(\sum_{i=1}^{n} C_{i}^{*} X_{i}^{-\lambda} C_{i}\right)^{\frac{\beta}{-\lambda}} \leq\left(\sum_{i=1}^{n} C_{i}^{*} X_{i}^{-\mu} C_{i}\right)^{\frac{\beta}{-\mu}}=g_{1}(-\mu)
$$

for $-\lambda \leq-\mu \leq-\beta<0$. Therefore $g_{1}(\lambda)$ is increasing for $\lambda$ such that $\lambda \leq-\beta$ or $\beta \leq \lambda$.

(ii) Taking logarithm to (2.4), we have

$$
g_{2}(\mu)=\frac{1}{\mu} \log \left(\sum_{i=1}^{n} C_{i}^{*} X_{i}^{\mu} C_{i}\right) \leq \frac{1}{\lambda} \log \left(\sum_{i=1}^{n} C_{i}^{*} X_{i}^{\lambda} C_{i}\right)=g_{2}(\lambda)
$$

for $0<\mu \leq \lambda$. By Theorem 2.A,

$$
g_{2}(\mu)=\frac{1}{\mu} \log \left(\sum_{i=1}^{n} C_{i}^{*} X_{i}^{\mu} C_{i}\right) \geq \frac{1}{\mu} \sum_{i=1}^{n} C_{i}^{*}\left(\log X_{i}^{\mu}\right) C_{i}=\sum_{i=1}^{n} C_{i}^{*}\left(\log X_{i}\right) C_{i}=g_{2}(0)
$$

since $f(x)=\log x$ is operator concave. Therefore $g_{2}(0) \leq g_{2}(\mu) \leq g_{2}(\lambda)$ for $0<\mu \leq \lambda$. We also get $g_{2}(-\lambda) \leq g_{2}(-\mu) \leq g_{2}(0)$ for $-\lambda \leq-\mu<0$ by replacing $X_{i}$ by $X_{i}^{-1}$. Hence the proof is complete.

Proof of Theorem 2.3. (i) By putting $X_{i}=A_{i}^{\frac{-1}{2}} B_{i} A_{i}^{\frac{-1}{2}}$ and $C_{i}=A_{i}^{\frac{1}{2}}$ in (i) in Lemma 2.4, we have that

$$
\left\{\sum_{i=1}^{n} A_{i}^{\frac{1}{2}}\left(A_{i}^{\frac{-1}{2}} B_{i} A_{i}^{\frac{-1}{2}}\right)^{t} A_{i}^{\frac{1}{2}}\right\}^{\frac{\beta}{t}}=\left(\sum_{i=1}^{n} A_{i} \bigsqcup_{t} B_{i}\right)^{\frac{\beta}{t}}
$$

is increasing for $t$ such that $t \leq-\beta$ or $\beta \leq t$.

Therefore we obtain that $K_{t, \frac{\beta}{t}}(\mathbb{A} \mid \mathbb{B})=\frac{\left(\sum_{i=1}^{n} A_{i} \natural_{t} B_{i}\right)^{\frac{\beta}{t}}-I}{\beta}$ is increasing for $t$ such that $t \leq-\beta$ or $\beta \leq t$.

(ii) Since $K_{t, \frac{-\beta}{t}}(\mathbb{A} \mid \mathbb{B})=\frac{\left\{\left(\sum_{i=1}^{n} A_{i} \natural_{t} B_{i}\right)^{\frac{\beta}{t}}\right\}^{-1}-I}{-\beta}$, we have (ii) by (2.6).

(iii) Similarly to (i), we obtain (iii) by using (ii) in Lemma 2.4.

By Proposition 2.2 and Theorem 2.3, we can obtain monotonicity of $K_{t, s}(\mathbb{A} \mid \mathbb{B})$ on $t$.

Theorem 2.5. Let $\mathbb{A}=\left(A_{1}, \cdots, A_{n}\right)$ and $\mathbb{B}=\left(B_{1}, \cdots, B_{n}\right)$ be operator distributions. 
(i) For $0 \leq s \leq 1, K_{t, s}(\mathbb{A} \mid \mathbb{B})$ is increasing for $t \in \mathbb{R}$.

(ii) $T_{t}(\mathbb{A} \mid \mathbb{B})$ is increasing for $t \in \mathbb{R}$.

Proof. (i) We may assume $0<s \leq 1$ since $K_{t, 0}(\mathbb{A} \mid \mathbb{B})=I_{t}(\mathbb{A} \mid \mathbb{B})$ by (i) in Proposition 2.1 and $I_{t}(\mathbb{A} \mid \mathbb{B})$ is increasing for $t \in \mathbb{R}$ by (iii) in Theorem 2.3.

If $0<t \leq u$, then

$$
K_{t, s}(\mathbb{A} \mid \mathbb{B}) \leq K_{u, \frac{t s}{u}}(\mathbb{A} \mid \mathbb{B}) \leq K_{u, s}(\mathbb{A} \mid \mathbb{B})
$$

holds by (i) in Theorem 2.3 and (i) in Proposition 2.2 since $0<t s \leq t \leq u$.

If $u \leq t<0$, then

$$
K_{u, s}(\mathbb{A} \mid \mathbb{B}) \leq K_{u, \frac{t s}{u}}(\mathbb{A} \mid \mathbb{B}) \leq K_{t, s}(\mathbb{A} \mid \mathbb{B})
$$

holds by (ii) in Proposition 2.2 and (ii) in Theorem 2.3 since $u \leq t \leq t s<0$.

By letting $t \rightarrow+0$ in (2.7) and $t \rightarrow-0$ in (2.8), we have

$$
K_{v, s}(\mathbb{A} \mid \mathbb{B}) \leq K_{0, s}(\mathbb{A} \mid \mathbb{B})=S(\mathbb{A} \mid \mathbb{B}) \leq K_{u, s}(\mathbb{A} \mid \mathbb{B})
$$

for $v<0<u$ by (iii) in Proposition 2.2. Therefore $K_{t, s}(\mathbb{A} \mid \mathbb{B})$ is increasing for $t \in \mathbb{R}$.

(ii) We have (ii) by putting $s=1$ in (i), or immediately obtain since for $a>0$,

$$
f(t)= \begin{cases}\frac{a^{t}-1}{t} & (t \neq 0) \\ \log a & (t=0)\end{cases}
$$

is increasing for $t \in \mathbb{R}$.

\section{Shannon TyPe inequalities}

In this section, we get several Shannon type inequalities. First, we obtain an extension of (1.1) and (1.2).

Theorem 3.1. Let $\mathbb{A}=\left(A_{1}, \cdots, A_{n}\right)$ and $\mathbb{B}=\left(B_{1}, \cdots, B_{n}\right)$ be operator distributions.

(i) For $0<\beta \leq t \leq 1$,

$$
\begin{aligned}
& K_{-1,-\beta}(\mathbb{A} \mid \mathbb{B}) \leq K_{-t, \frac{\beta}{-t}}(\mathbb{A} \mid \mathbb{B}) \leq K_{-\beta,-1}(\mathbb{A} \mid \mathbb{B}) \\
\leq & T_{\beta}(\mathbb{A} \mid \mathbb{B}) \leq K_{t, \frac{\beta}{t}}(\mathbb{A} \mid \mathbb{B}) \leq 0 .
\end{aligned}
$$

(ii) For $0<\beta \leq t \leq 1$,

$$
\begin{aligned}
K_{-1, \beta}(\mathbb{A} \mid \mathbb{B}) & \leq K_{-t, \frac{\beta}{t}}(\mathbb{A} \mid \mathbb{B}) \leq T_{-\beta}(\mathbb{A} \mid \mathbb{B}) \\
\leq K_{\beta,-1}(\mathbb{A} \mid \mathbb{B}) & \leq K_{t, \frac{-\beta}{t}}(\mathbb{A} \mid \mathbb{B}) \leq 0
\end{aligned}
$$

(iii) For $0<t \leq 1$,

$$
I_{-1}(\mathbb{A} \mid \mathbb{B}) \leq I_{-t}(\mathbb{A} \mid \mathbb{B}) \leq S(\mathbb{A} \mid \mathbb{B}) \leq I_{t}(\mathbb{A} \mid \mathbb{B}) \leq 0
$$


We remark that (iii) in Theorem 3.1 implies (1.1) since

$$
I_{-1}(\mathbb{A} \mid \mathbb{B})=-\log \left[\sum_{i=1}^{n} A_{i} B_{i}^{-1} A_{i}\right]
$$

and also (i) in Theorem 3.1 implies (1.2) since

$$
K_{-1,-\beta}(\mathbb{A} \mid \mathbb{B})=\frac{\left(\sum_{i=1}^{n} A_{i} B_{i}^{-1} A_{i}\right)^{-\beta}-I}{\beta} .
$$

Proof of Theorem 3.1. (i) By (i) in Theorem 2.3, we have

$$
K_{-1,-\beta}(\mathbb{A} \mid \mathbb{B}) \leq K_{-t, \frac{\beta}{-t}}(\mathbb{A} \mid \mathbb{B}) \leq K_{-\beta,-1}(\mathbb{A} \mid \mathbb{B})
$$

and

$$
T_{\beta}(\mathbb{A} \mid \mathbb{B})=K_{\beta, 1}(\mathbb{A} \mid \mathbb{B}) \leq K_{t, \frac{\beta}{t}}(\mathbb{A} \mid \mathbb{B}) \leq K_{1, \beta}(\mathbb{A} \mid \mathbb{B})=0 .
$$

Since $f(x)=-x^{-1}$ is operator concave, by Theorem 2.A, we have

$$
\begin{aligned}
K_{-\beta,-1}(\mathbb{A} \mid \mathbb{B}) & =\frac{\left(\sum_{i=1}^{n} A_{i} \natural_{-\beta} B_{i}\right)^{-1}-I}{\beta}=\frac{\left\{\sum_{i=1}^{n} A_{i}^{\frac{1}{2}}\left(A_{i}^{\frac{-1}{2}} B_{i} A_{i}^{\frac{-1}{2}}\right)^{-\beta} A_{i}^{\frac{1}{2}}\right\}^{-1}-I}{\beta} \\
& \leq \frac{\sum_{i=1}^{n} A_{i}^{\frac{1}{2}}\left(A_{i}^{\frac{-1}{2}} B_{i} A_{i}^{\frac{-1}{2}}\right)^{\beta} A_{i}^{\frac{1}{2}}-I}{\beta}=T_{\beta}(\mathbb{A} \mid \mathbb{B}) .
\end{aligned}
$$

Therefore the desire inequality is obtained.

(ii) is shown by the similar way to the proof of (i).

(iii) holds by (iii) in Theorem 2.3, or we can get (iii) by letting $\beta \rightarrow+0$ in (i) or (ii).

Combining Theorem 3.1 and the results shown in section 2, we have the following Theorem 3.2. We remark that (i) in Theorem 3.2 is a generalization of (2.1).

Theorem 3.2. Let $\mathbb{A}=\left(A_{1}, \cdots, A_{n}\right)$ and $\mathbb{B}=\left(B_{1}, \cdots, B_{n}\right)$ be operator distributions.

(i) For $0<\beta \leq t \leq 1$ and $0<s<1$,

$$
\begin{aligned}
& K_{-1, \beta}(\mathbb{A} \mid \mathbb{B}) \leq K_{-t, \frac{\beta}{t}}(\mathbb{A} \mid \mathbb{B}) \leq T_{-\beta}(\mathbb{A} \mid \mathbb{B}) \leq K_{-\beta, s}(\mathbb{A} \mid \mathbb{B}) \leq I_{-\beta}(\mathbb{A} \mid \mathbb{B}) \\
\leq & S(\mathbb{A} \mid \mathbb{B}) \leq I_{\beta}(\mathbb{A} \mid \mathbb{B}) \leq K_{\beta, s}(\mathbb{A} \mid \mathbb{B}) \leq T_{\beta}(\mathbb{A} \mid \mathbb{B}) \leq K_{t, \frac{\beta}{t}}(\mathbb{A} \mid \mathbb{B}) \leq 0 .
\end{aligned}
$$

(ii) For $0<t \leq 1$ and $0<s \leq 1$,

$$
K_{-t,-s}(\mathbb{A} \mid \mathbb{B}) \leq K_{t, s}(\mathbb{A} \mid \mathbb{B}) \leq 0 \text { and } K_{-t, s}(\mathbb{A} \mid \mathbb{B}) \leq K_{t,-s}(\mathbb{A} \mid \mathbb{B}) \leq 0
$$

(iii) For $0<\beta \leq 1$,

$$
I-\sum_{i=1}^{n} A_{i} B_{i}^{-1} A_{i} \leq K_{-1, \beta}(\mathbb{A} \mid \mathbb{B}) \leq I_{-1}(\mathbb{A} \mid \mathbb{B}) \leq K_{-1,-\beta}(\mathbb{A} \mid \mathbb{B}) \leq T_{\beta}(\mathbb{A} \mid \mathbb{B}) \leq 0 .
$$


Proof. (i) We get

and

$$
T_{\beta}(\mathbb{A} \mid \mathbb{B}) \leq K_{t, \frac{\beta}{t}}(\mathbb{A} \mid \mathbb{B}) \leq 0
$$

$$
K_{-1, \beta}(\mathbb{A} \mid \mathbb{B}) \leq K_{-t, \frac{\beta}{t}}(\mathbb{A} \mid \mathbb{B}) \leq T_{-\beta}(\mathbb{A} \mid \mathbb{B})
$$

by (i) and (ii) in Theorem 3.1. The rest holds by (iii) and (iv) in Proposition 2.1.

(ii) By putting $\beta=t s$ in (i) and (ii) in Theorem 3.1, we have (ii).

(iii) By (ii) in Proposition 2.2, we have

$$
K_{-1,1}(\mathbb{A} \mid \mathbb{B}) \leq K_{-1, \beta}(\mathbb{A} \mid \mathbb{B}) \leq K_{-1,0}(\mathbb{A} \mid \mathbb{B}) \leq K_{-1,-\beta}(\mathbb{A} \mid \mathbb{B})
$$

that is,

$$
I-\sum_{i=1}^{n} A_{i} B_{i}^{-1} A_{i} \leq K_{-1, \beta}(\mathbb{A} \mid \mathbb{B}) \leq I_{-1}(\mathbb{A} \mid \mathbb{B}) \leq K_{-1,-\beta}(\mathbb{A} \mid \mathbb{B})
$$

for $0<\beta \leq 1$. The rest holds by (i) in Theorem 3.1.

\section{Operator VAlued $\alpha$-Divergence AND \\ SHANNON-NAKAMURA-UMEGAKI OPERATOR ENTROPY}

In this section, we state two topics related to relative operator entropies.

Based on $\alpha$-divergence of two probability distributions by Amari [1], Fujii [3] defined an operator valued $\alpha$-divergence as follows: For strictly positive operators $A$ and $B$, and for $\alpha \in(0,1)$,

$$
D_{\alpha}(A \mid B) \equiv \frac{1}{\alpha(1-\alpha)}\left(A \nabla_{\alpha} B-A \sharp_{\alpha} B\right)=\frac{1}{1-\alpha}\left\{B-A-T_{\alpha}(A \mid B)\right\},
$$

where $A \nabla_{\alpha} B \equiv(1-\alpha) A+\alpha B$ is the weighted arithmetic mean. Fujii-MićićPečarić-Seo $[5,6]$ showed

$$
\begin{aligned}
& D_{0}(A \mid B) \equiv \lim _{\alpha \rightarrow 0} D_{\alpha}(A \mid B)=B-A-S(A \mid B) \text { and } \\
& D_{1}(A \mid B) \equiv \lim _{\alpha \rightarrow 1} D_{\alpha}(A \mid B)=A-B+S_{1}(A \mid B) .
\end{aligned}
$$

We remark that $D_{0}(A \mid B)$ is a divergence introduced by Petz [15]. It is easily seen that $D_{1}(A \mid B)=D_{0}(B \mid A)$ since we can show $S_{1}(A \mid B)=-S(B \mid A)$ by using an equation $f\left(X X^{*}\right) X=X f\left(X^{*} X\right)$ for a continuous function $f$ on an interval $J$ and an operator $X$ such that spectra of $X X^{*}$ and $X^{*} X$ are contained in $J$.

In [11], we define operator valued $\alpha$-divergence $D_{\alpha}(\mathbb{A} \mid \mathbb{B})$ of two operator distributions $\mathbb{A}=\left(A_{1}, \cdots, A_{n}\right)$ and $\mathbb{B}=\left(B_{1}, \cdots, B_{n}\right)$ as

$$
D_{\alpha}(\mathbb{A} \mid \mathbb{B}) \equiv \sum_{i=1}^{n} D_{\alpha}\left(A_{i} \mid B_{i}\right)
$$

We note that it was shown in [11] that

$$
\begin{aligned}
& D_{\alpha}(\mathbb{A} \mid \mathbb{B})=\frac{-1}{1-\alpha} T_{\alpha}(\mathbb{A} \mid \mathbb{B}) \text { for } \alpha \in(0,1) \\
& D_{0}(\mathbb{A} \mid \mathbb{B})=-S(\mathbb{A} \mid \mathbb{B}) \text { and } D_{1}(\mathbb{A} \mid \mathbb{B})=D_{0}(\mathbb{B} \mid \mathbb{A})=S_{1}(\mathbb{A} \mid \mathbb{B}),
\end{aligned}
$$


so that $(2.1)$ is equivalent to

$$
0 \leq(1-\alpha) D_{\alpha}(\mathbb{A} \mid \mathbb{B}) \leq-K_{t, s}(\mathbb{A} \mid \mathbb{B}) \leq-I_{t}(\mathbb{A} \mid \mathbb{B}) \leq D_{0}(\mathbb{A} \mid \mathbb{B})
$$

for $0<t \leq 1$ and $0<s<1$.

Next, as an operator version of Shannon entropy and as an expression of Nakamura-Umegaki operator entropy $H(A)$ by using an operator distribution, we introduce Shannon-Nakamura-Umegaki operator entropy of an operator distribution $\mathbb{A}=\left(A_{1}, \cdots, A_{n}\right)$ as

$$
H(\mathbb{A}) \equiv \sum_{i=1}^{n} H\left(A_{i}\right)=\sum_{i=1}^{n} A_{i} \log A_{i}^{-1}
$$

Then the following fundamental properties hold.

Proposition 4.1. Let $\mathbb{A}=\left(A_{1}, \cdots, A_{n}\right)$ be an operator distribution and $\frac{1}{n} \mathbb{I}=$ $\left(\frac{1}{n} I, \cdots, \frac{1}{n} I\right)$.

(i) $S\left(\mathbb{A} \mid \frac{1}{n} \mathbb{I}\right)=H(\mathbb{A})-(\log n) I$.

(ii) $0 \leq H(\mathbb{A}) \leq(\log n) I$.

Proof. (i) Since $S\left(A_{i} \mid \frac{1}{n} I\right)=A_{i} \log \left(A_{i}^{-1} \cdot \frac{1}{n} I\right)=A_{i} \log A_{i}^{-1}-(\log n) A_{i}$ holds for $i=1, \cdots, n$,

$$
S\left(\mathbb{A} \mid \frac{1}{n} \mathbb{I}\right)=\sum_{i=1}^{n}\left\{A_{i} \log A_{i}^{-1}-(\log n) A_{i}\right\}=H(\mathbb{A})-(\log n) I .
$$

(ii) We have $H(\mathbb{A}) \geq 0$ since $-x \log x \geq 0$ for $0<x \leq 1$ and the spectrum of $A_{i}$ is included in $(0,1]$ for $i=1, \cdots, n$. We have $H(\mathbb{A}) \leq(\log n) I$ by $(1.1)$ and (i).

Remark. In the proof of (ii) in Proposition 4.1, by using (2.1) instead of (1.1), we have

$$
\begin{aligned}
0 & \leq H(\mathbb{A}) \leq \frac{1}{t} \log \sum_{i=1}^{n} A_{i}^{1-t} \leq \frac{1}{t s}\left\{\frac{1}{n^{t s}}\left(\sum_{i=1}^{n} A_{i}^{1-t}\right)^{s}+\left(\log n^{t s}-1\right) I\right\} \\
& \leq \frac{1}{t}\left\{\frac{1}{n^{t}} \sum_{i=1}^{n} A_{i}^{1-t}+\left(\log n^{t}-1\right) I\right\} \leq(\log n) I
\end{aligned}
$$

for $0<t \leq 1$ since $A_{i} \bigsqcup_{t}\left(\frac{1}{n} I\right)=\frac{1}{n^{t}} A_{i}^{1-t}$ holds for $i=1, \cdots, n$. 


\section{REFERENCES}

1. S. Amari, Differential Geometrical Methods in Statistics, Springer Lecture Notes in Statistics, 28, 1985.

2. T. Furuta, Parametric extensions of Shannon inequality and its reverse one in Hilbert space operators, Linear Algebra Appl. 381 (2004), 219-235.

3. J.I. Fujii, On the relative operator entropy (in Japanese), RIMS Kokyuroku 903 (1995), $49-56$.

4. J.I. Fujii and E. Kamei, Relative operator entropy in noncommutative information theory, Math. Japon. 34 (1989), 341-348.

5. J.I. Fujii, J. Mićić, J. Pečarić and Y. Seo, Comparison of operator mean geodesics, J. Math. Inequal. 2(2008), 287-298.

6. M. Fujii, J. Mićić, J. Pečarić and Y. Seo, Recent Development of Mond-Pečarić Method in Operator Inequalities, Monographs in Inequalities 4, Element, Zagreb, (2012).

7. F. Hansen and G.K. Pedersen, Jensen's operator inequality, Bull. London Math. Soc. 35 (2003), 553-564.

8. H. Isa, M. Ito, E. Kamei, H. Tohyama and M. Watanabe, Relative operator entropy, operator divergence and Shannon inequality, Sci. Math. Jpn., 75 (2012), 289-298. (online: e-2012 (2012), 353-362.)

9. H. Isa, M. Ito, E. Kamei, H. Tohyama and M. Watanabe, Extensions of Tsallis relative operator entropy and operator valued distance, Sci. Math. Jpn. 76 (2013), 427-435. (online: e-2013 (2013), 427-435.)

10. H. Isa, M. Ito, E. Kamei, H. Tohyama and M. Watanabe, Generalizations of operator Shannon inequality based on Tsallis and Rényi relative entropies, Linear Algebra Appl. 439 (2013), 3148-3155.

11. H. Isa, M. Ito, E. Kamei, H. Tohyama and M. Watanabe, On relations between operator valued $\alpha$-divergence and relative operator entropies, Sci. Math. Jpn. (to appear).

12. S. Kullback, Information Theory and Statistics, Wiley, New York, 1959.

13. M. Nakamura and H. Umegaki, A note on the entropy for operator algebras, Proc. Japan Acad. 37 (1961), 149-154.

14. M.I. Nielsen and I.L. Chuang, Quantum Computation and Quantum Information, Cambridge University Press, Cambridge, 2000.

15. D. Petz, Bregman divergence as relative operator entropy, Acta Math. Hungar. 116 (2007), 127-131.

16. K. Yanagi, K. Kuriyama and S. Furuichi, Generalized Shannon inequalities based on Tsallis relative operator entropy, Linear Algebra Appl. 394 (2005), 109-118.

1 Maebashi Institute of Technology, 460-1 Kamisadorimachi, Maebashi, Gunma 371-0816, JAPAN.

E-mail address: isa@maebashi-it.ac.jp; m-ito@maebashi-it.ac.jp;

tohyama@maebashi-it.ac.jp; masayukiwatanabe@maebashi-it.ac.jp

2 1-1-3, Sakuragaoka, Kanmakicho, Kitakaturagi-gun, Nara 639-0202, Japan. E-mail address: ekamei1947@yahoo.co.jp 